\title{
Los estudios métricos de información en Brasil y en España a partir de los artículos recogidos en la WoS
}

\author{
María Luisa Lascurain \\ Doutora; Universidad Carlos III de Madrid, Madrid, España; \\ mlascura@bib.uc3m
}

\begin{abstract}
Resumen:
Este trabajo analiza la presencia de artículos sobre las disciplinas que forman los Estudios Métricos de Información (EMI) en la WoS, firmados por autores de Brasil y de España. Se parte de un número muy bajo de documentos que aumenta a lo largo del tiempo, con un CMA superior para Brasil $(25,17 \%)$ que para España (13,57\%). El idioma principal, con aproximadamente el $60 \%$ de los artículos, es el inglés. La temática, examinada a partir de las categorías WoS y de la agrupación propuesta por Glänzel y Schubert (2003), indica la existencia de otras áreas diferentes a LIS, como Ingeniería o Medicina. La interdisciplinariedad de los trabajos se manifiesta también en las revistas de publicación. El núcleo de revistas lo forman revistas nacionales (el 72,73\% para Brasil y el 50\% para España). La colaboración entre los países es muy escasa, con solo nueve registros en todo el periodo de estudio.
\end{abstract}

Palabras clave: Estudios métricos de información. Análisis bibliométrico. Brasil. España.

\section{Introducción}

Los estudios métricos de información (EMI), constituyen un campo científico que agrupa diversas disciplinas dirigidas a la cuantificación de los distintos aspectos vinculados a la información y a sus usuarios, y que tienen como objetivos el análisis e evaluación de los procesos relacionados con la producción, difusión y consumo de información y de las regularidades de los flujos de información científica. Como señala Gorbea-Portal (2013, p. 16), “[...] constituyen un campo multi, inter y transdiscipliario en el cual interactúan diversos métodos y modelos matemáticos y estadísticos con las disciplinas que integran el denominado Sistema de Conocimientos Bibliológico Informativo".

Entre las disciplinas que forman los EMI, la Bibliometría es pionera a partir de los análisis estadísticos que con una rigurosa metodología se dirigían a 
conocer las características de los documentos publicados sobre determinados temas como los de Cole y Eales (1917), sobre anatomía comparada o de Hulme (1923), sobre historia de la Ciencia. Además destaca la investigación publicada sobre las regularidades que afectan a la comunicación científica, las denominadas "leyes bibliométricas" vinculadas a la distribución de las palabras en los textos o Ley de Zipf, (1926), la Ley de Lotka sobre la productividad científica de los investigadores de ese mismo año, la distribución de los artículos en las revistas, la llamada Ley de Bradford (1934) o sobre la obsolescencia de la literatura científica. Sin embargo el mayor crecimiento de la Bibliometría se produce a partir de la década de los 70 del siglo $\mathrm{XX}$, con importantes transformaciones de la mano del desarrollo de las tecnologías de la información y de las comunicaciones.

La Cienciometría, surgió como área de investigación en Europa Oriental, concretamente en Hungría donde se desarrollaron un gran número de trabajos de esta materia, y se creó la revista Scientometrics, en 1978, editada por la Academia de Ciencias.

Con base similar a ambas disciplinas, surge la Informetría, definida por primera vez por Otto Nacke (1979) y que obtuvo un reconocimiento fundamental entre la comunidad científica cuando el Encuentro Internacional de Bibliometría pasó a denominarse, en 1989 Conferencia Internacional de Bibliometría, Cienciometría e Informetría.

Por su parte, la Patentometría permite conocer las relaciones entre ciencia y tecnología y las características de la innovación patentada. Como señalan Hidalgo-Nuchera; Iglesias-Pradas e Hernández-García (2009), a partir de los primeros trabajos que utilizaron las patentes como indicadores de actividades tecnológicas a mediados de los años sesenta, se ha incrementado cuantitativa y cualitativamente su utilización para el análisis de los procesos de innovación y de las capacidades tecnológicas de países, instituciones, empresas y particulares.

En cuanto a los estudios de usuarios, si bien no proceden del mismo tronco que las disciplinas anteriores, comparten métodos y objetivos y son muchas las sinergias entre ellas, especialmente con la Bibliometría, ya que 
muchos autores han dedicado su investigación a los dos aspectos de la comunicación científica, la producción y el consumo de información.

La Cibermetría y la Webmetría surgen a mediados de los años 1990, con la aplicación de los supuestos y metodología de los EMI que las precedieron aplicados a internet.

La última disciplina surgida en el ámbito de los EMI es la Altmetría, que se plantea como una "métrica alternativa" cuya investigación se centra fundamentalmente en la comparación con indicadores métricos tradicionales y en la identificación de las herramientas 2.0 de mayor utilidad en el análisis métrico de la información.

En España, el inicio de la actividad bibliométrica arranca a principios de los años 70, de la mano de López Piñero y desde entonces:

[...] se ha extendido e implantado como técnica metodológica imprescindible para la evaluación de la producción científica y de todos los fenómenos ligados a la comunicación de la ciencia. Su importancia ha sido capital no sólo para el desarrollo de la Biblioteconomía y Documentación como disciplina actividad profesional científica en España. (DELGADO LÓPEZ-COZAR, 2002, p. 4).

De hecho, son varios los estudios que ponen de manifiesto la aportación de los autores que se dedican a distintas especialidades métricas al conjunto de la investigación en Información y Documentación en España (ALCAÍNPARTEARROYO, 1991; LÓPEZ-PIÑERO; TERRADA， 1993; FRÍAS; ROMERO, 1998; ARQUERO-AVILÉS; SALVADOR-OLIVÁN; ANTONIO, 2004; DELGADO LÓPEZ-COZAR et al., 2006; ARGUIMBAU-VIVÓ; FUENTES-PUJOL; GALLIFA-CALATAYUD, 2013).

Según Urbizagástegui-Alvarado (1984) el inicio de la Bibliometría en Brasil está vinculado al curso de Maestría en Ciencia de la Información del IBICT de 1970 y establece el surgimiento de los primeros trabajos con metodología bibliométrica en 1972 y 1974, con una importante presencia hasta los años 80 de los temas relacionados con la dispersión de la literatura científica, por su interés en la aplicación a la gestión bibliotecaria. Vanz (2003) destaca el escaso número de publicaciones sobre bibliometría hasta finales de la década de 
los 90 y señala las entre las causas de un posterior crecimiento de los EMI en Brasil la creación de la base SciELO, que requirió el conocimiento de la producción científica nacional de estudios bibliométricos y cienciométricos. También la mayor difusión de las bases de datos del ISI (Institute for Scientific Information) a través de la plataforma WoS disponible en las principales universidades y centros de investigación brasileños. Además CAPES potenció los estudios bibliométricos en su labor de evaluación de la actividad científica de universidades e investigadores así como de su impacto y visibilidad internacional.

Por su parte Meis y Leta (1996 apud VANZ, 2003), consideran que los estudios en el campo de la Cienciometría en Brasil comenzaron en 1977 con un trabajo publicado por Morel y Morel sobre la producción científica brasileña en las bases de datos del ISI.

El objetivo de este trabajo es caracterizar la producción científica recogida en la WoS firmada por autores de Brasil y de España sobre estudios métricos de información, determinando sus similitudes y diferencias en cuanto a producción total de artículos y su evolución a lo largo del tiempo, idioma, temática y revistas de publicación, así como las singularidades de los trabajos en colaboración hispano-brasileña.

\section{Metodología}

La utilización de la Web of Science (WoS) como fuente para la recuperación de los registros se justifica por la importancia de este recurso como referente de la difusión científica internacional y por la ventaja que supone el que incluya la dirección institucional de todos los autores firmantes. No obstante, se tienen en consideración las limitaciones y sesgos que presenta en cuanto a cobertura de países no anglosajones, a favor del inglés y de los artículos frente a otros tipos documentales o la sobrerrepresentación de las Ciencias Puras y Experimentales frente a las Ciencias Sociales y, en mayor medida, las Humanidades (GÓMEZ; BORDONS, 1996; TORRES-SALINAS et al., 2010; ARCHAMBAULT; LARIVIÈRE, 2010; DELGADO-LÓPEZ COZAR et al., 2014). 
Se consultaron (julio de 2015) las tres bases de datos de la colección principal de la WoS (SCIExpanded, SSCI y A\&HCI) según la estrategia de búsqueda que recuperaría los registros relacionados con estudios métricos de información: en el campo tema (TS) los términos que nombran las categoría que conforma los EMI (bibliometr*, informetr*, scientometr*, patentometr*, webometr*, webmetr*, cybermetrc*, almetric*) además de "user studies" y "metrics studies" combinados con la categoría Information Science and Library Science para evitar el ruido que se producen al buscarlos de forma aislada en el campo tema (TS).

Los resultados obtenidos se combinaron con los de la búsqueda de la producción científica de cada país y de los documentos escritos en colaboración (CU=Spain/ CU= Brazil/ CU=Spain and Brazil).

Se eliminaron los registros correspondientes al año 2015, sin ninguna otra delimitación temporal para poder así identificar el surgimiento de los primeros trabajos sobre el tema de estudio en los países seleccionados.

La tipología documental quedó restringida a los artículos dada su importancia frente a otros tipos documentales como medio de difusión de la información científica (GARVEY; GRIFFITH, 1972) y para dar homogeneidad a la muestra. Por otra parte, su presencia frente a otros tipos documentales explica esta selección, ya que supone el 90,96\% de las publicaciones españolas y el $86,90 \%$ de las de Brasil.

Se utilizaron indicadores bibliométricos unidimensionales y multidimensionales para analizar las características de los artículos seleccionados. Entre los primeros, los indicadores relativos a la producción científica y a su evolución temporal, idioma de publicación y temática. Esta se ha obtenido a partir de las categorías WoS y de la clasificación propuesta por Glänzel y Schubert (2003) que agrupa las categorías WoS en 14 grandes áreas, 12 correspondientes al SCI y dos relativas al SSCI y al A\&HCI. La categoría Information Science \& Library Science se consideró por separado, desagregándola de Social Sciences, por su valor en el estudio, donde el 83,45\% de los trabajos de sobre Ciencias Sociales corresponden a esta materia en el caso de España y el $72,73 \%$ en Brasil. Para conocer las principales revistas de 
publicación se aplicó el modelo propuesto por Bradford, considerando los títulos que recogen la mitad de los trabajos de cada país. Estos indicadores se han aplicado a las muestras de Brasil, de España y de los artículos en que colaboran estos países.

Para el estudio de las relaciones entre las variables fecha de publicación y temática (según áreas Glänzel y Schubert) se ha empleado el análisis de correspondencias (AC) y la representación gráfica obtenida a partir de este que facilita la interpretación de dicha información.

\section{Resultados}

En esta sección se presentan los resultados.

\subsection{Producción científica en la WoS sobre EMI de España y Brasil.}

El total de artículos recogidos en la WoS firmados por autores adscritos a instituciones españolas es de 744, distribuidos a lo largo de 31 años, con un promedio de 24 artículos anuales. La figura 1 muestra la distribución temporal de los trabajos que se inicia en el año 1978 (con dos artículos) y continua con una presencia muy escasa hasta 1990. De hecho, si separamos en dos partes la producción, el promedio del primer periodo es de 4,25 artículos, mientras que en segundo alcanza los 45,07. El incremento se refleja en la línea de tendencia y en el valor de $r^{2}$ que indica una dependencia entre las variables con un porcentaje de cerca del 75\%. El crecimiento medio acumulativo (CMA) para los 31 años analizados es del 13,57\%, similar al del primer periodo (CMA=13,85\%), pero inferior al correspondiente al segundo $(\mathrm{CMA}=17,97 \%)$.

La variación interanual muestra valores negativos para nueve de los 31 años, siendo el valor más bajo el del año 1993 (-0,80). El valor positivo más alto (nueve) corresponde al año 1992. 
Figura 1 - Evolución temporal del número de artículos firmados por autores españoles. Variación interanual.

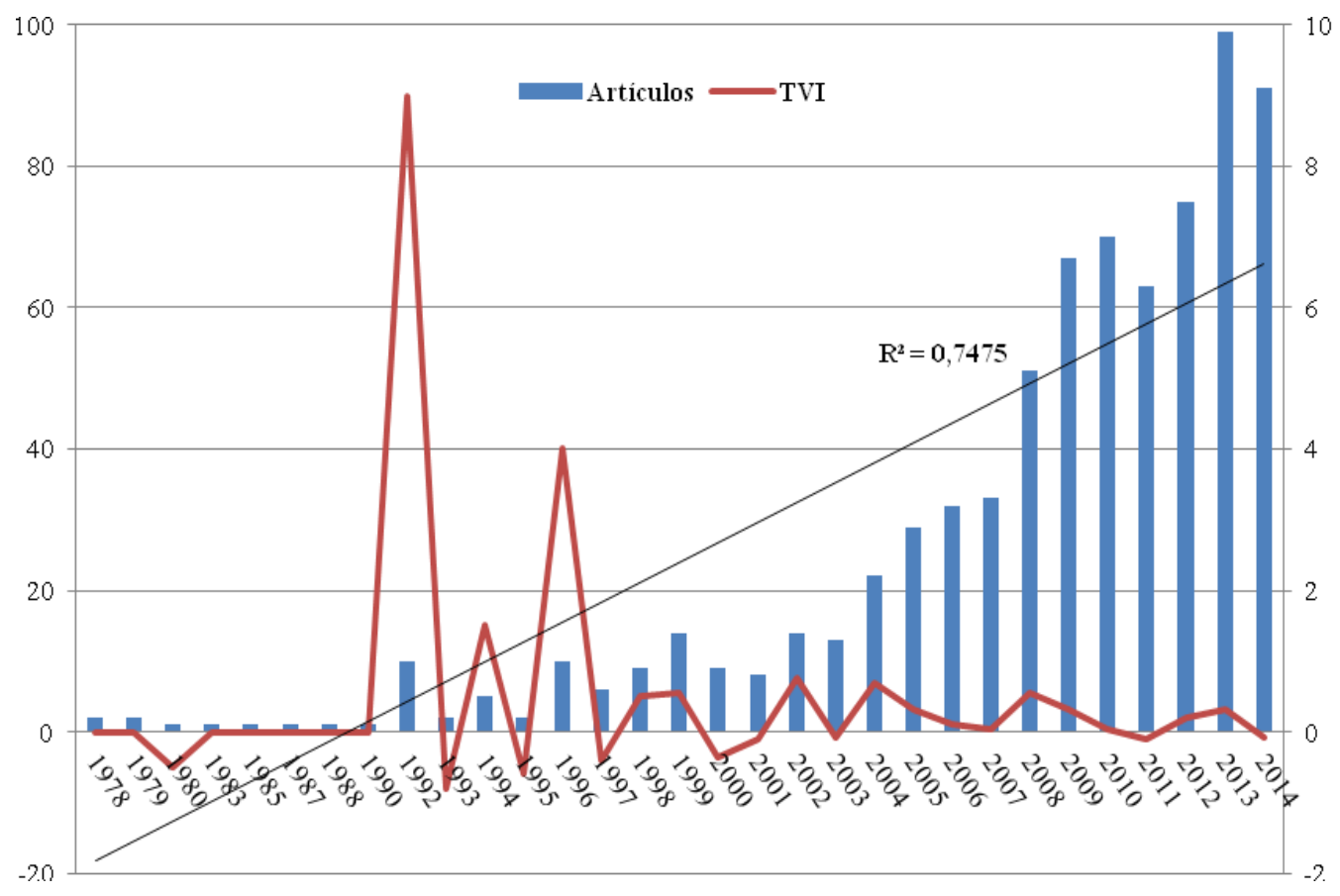

Fuente: WoS. Elaboración propia

De los 219 artículos recuperados de Brasil a lo largo de 16 años, el primero corresponde a 1991. El promedio es de 13,69 artículos anuales, pero como en el caso de España, la distribución (recogida en la figura 2) muestra un crecimiento destacado a partir de la segunda mitad con un promedio de 24 artículos anuales, ocho veces superior al de la primera $(3,38)$. En cuanto al crecimiento medio acumulativo (CMA) para el conjunto de la muestra, es del $25,17 \%$.

La variación interanual muestra valores negativos en cinco años y valores positivos más bajos que la muestra de España. 
Figura 2 - Evolución temporal del número de artículos firmados por autores brasileños. Variación interanual.

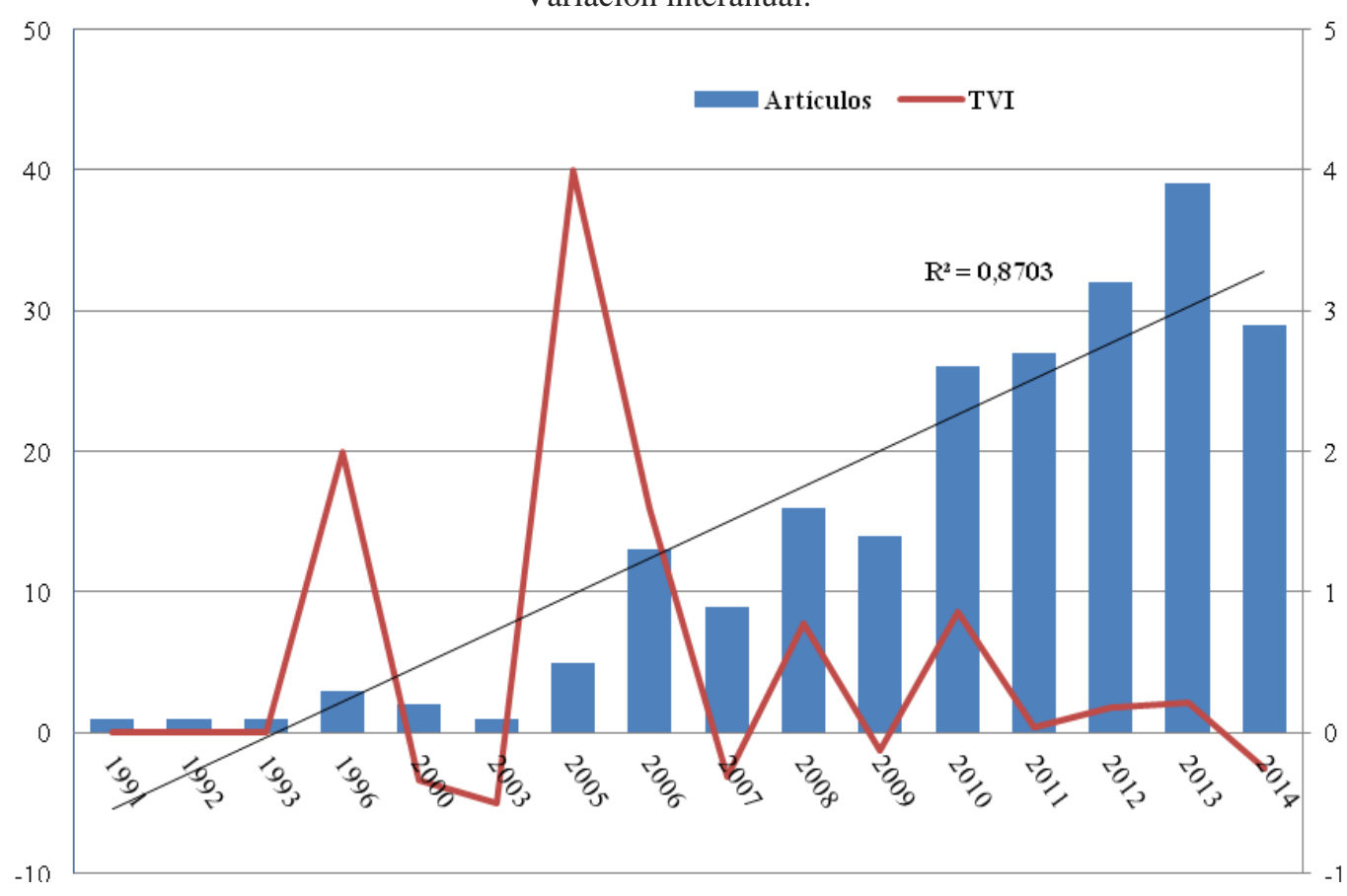

Fuente: WoS. Elaboración propia

Con respecto al idioma de publicación, la tabla 1 muestra para ambos países el uso del inglés en el $60 \%$ de sus publicaciones, mientras que el porcentaje de documentos en la lengua materna oscila entre el 38\% (ESP) y el $35 \%$ (BRA). Los autores brasileños publican un 5\% en español mientras que, a la inversa, el porcentaje es menor, solo el 1,21\% de los artículos firmados por españoles está escrito en portugués.

Tabla 1 - Idioma de publicación de los artículos (España y Brasil)

\begin{tabular}{|c|c|c|c|c|}
\hline \multirow[b]{2}{*}{ Idioma } & \multicolumn{2}{|c|}{ España } & \multicolumn{2}{|c|}{ Brasil } \\
\hline & Frecuencia & $\%$ & Frecuencia & $\%$ \\
\hline Inglés & 452 & 60,75 & 131 & 59,82 \\
\hline Lengua materna & 283 & 38,04 & 77 & 35,16 \\
\hline Portugués & 9 & 1,21 & & \\
\hline Español & & & 11 & 5,02 \\
\hline Total & 744 & 100 & 219 & 100 \\
\hline
\end{tabular}

En un primer análisis de los temas según la asignación de categorías de la WoS, se observa que la mayor parte de los trabajos está asignado a 
Information Science \& Library Science (LIS) presente en el 48,12\% de los artículos de los autores de España y en el 36,53\% de los de Brasil, seguido de la categoría Computer Science, Interdisciplinary Applications (17,47 \% en España y $10,50 \%$ en Brasil).

En el resto de categorías se constatan diferencias entre los países en cuanto a temas. En Medicine, General \& Internal el porcentaje de trabajos de España (4,44\%) es el doble que el de Brasil (2,74\%), en Public, Environmental \& Occupational Health la diferencia es a favor de Brasil (9,59\% frente a 2,44 ESP) y Psychology, Multidisciplinary figura en el 4,44\% de trabajos en España, mientras que es una categoría que no aparece en los artículos firmados por los autores de Brasil. O en el caso contrario Biology, 4,94\% y cuarta en orden de preferencia entre los autores de Brasil y sin presencia en la muestra de España.

Otro análisis temático surge de la agregación de las disciplinas WoS en las áreas Glänzel y Schubert (2003) separando la categoría Information Science \& Library Science (LIS), como se indica en el apartado de metodología. LIS ocupa el primer lugar $(\mathrm{ESP}=48,12 \%, \mathrm{BRA}=36,57 \%)$ con una diferencia porcentual de 11,55 a favor de España. Entre las áreas más presentes en ambos figura Clinical and Experimental Medicine II (Non-Internal Medicine Specialties) con distintos porcentajes (23,19\% BRA y $13,17 \%$ ESP). Los temas que agrupa Engineering están presentes en el 31,45\% de las artículos españoles, frente al 16,44 de los brasileños. Otra diferencia destacada se produce en Neuroscience \& Behavior (ESP $=10,35 \%$ y quinta posición y $\mathrm{BRA}=0,91 \%$ ). (Figura 3). 
Figura 3 - Distribución de la producción española y brasileña según áreas Glänzel y Schubert.

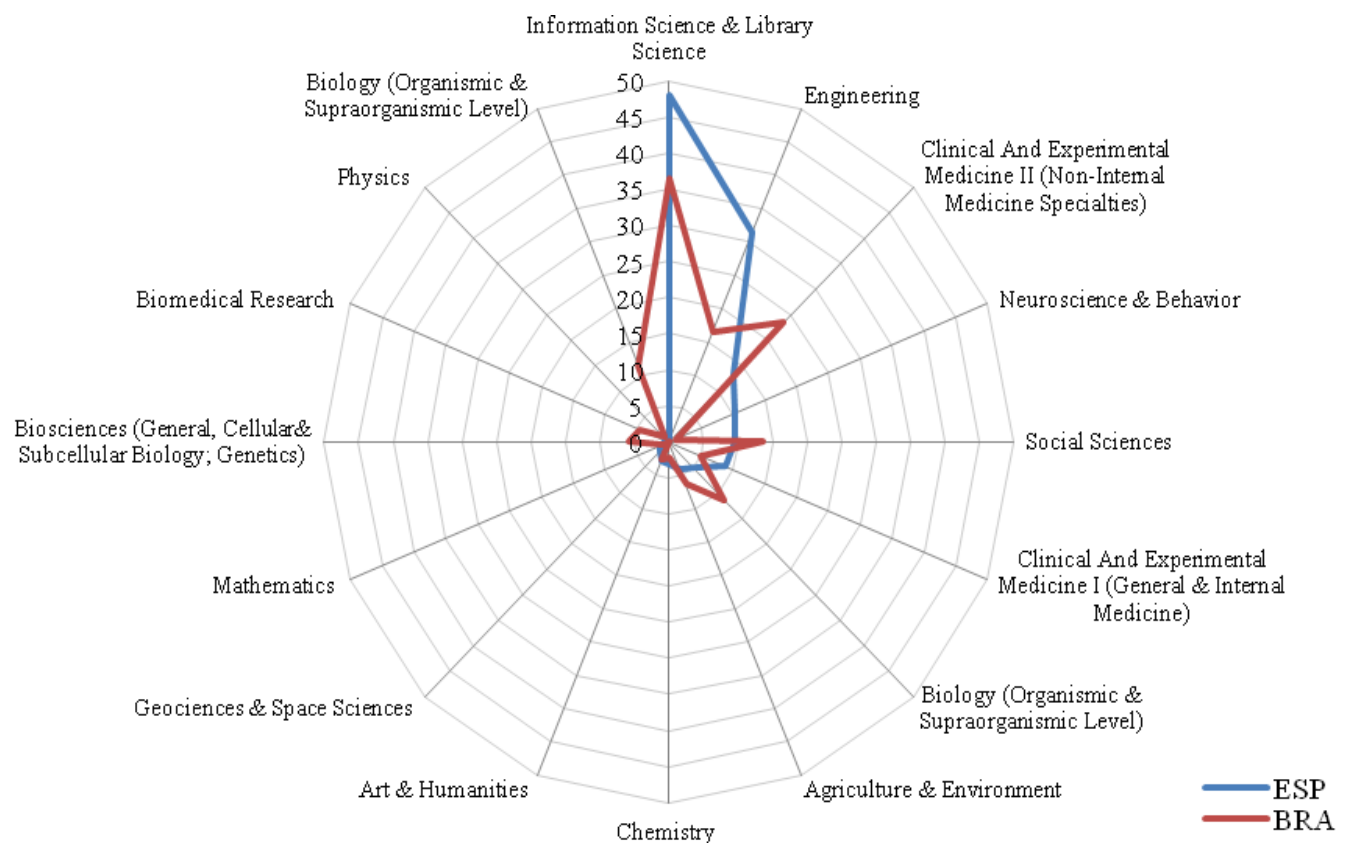

Fuente: WoS. Elaboración propia

Para mostrar la evolución temporal de los temas se ha realizado un análisis de correspondencias cuya representación gráfica se recoge en las figuras 4-5 (ESP) y 6 (BRA).

En los mapas de los países, aparece en el centro la categoría Information Science \& Library Science (LIS), puesto que como era de esperar los documentos sobre este tema están presentes a lo largo de todo el periodo estudiado. En el caso de España, la mayor parte de las áreas comparte este perfil poco específico en cuanto a distribución temporal, situándose también en el centro, con algunas excepciones como Physics (PHY) y Arts \&Humanities $(\mathrm{A} \& \mathrm{H})$ disciplina más presente a mediados y finales de los años 80 (Fig. 4). 
Figura 4 - Análisis de correspondencias año de publicación/área temática (España).

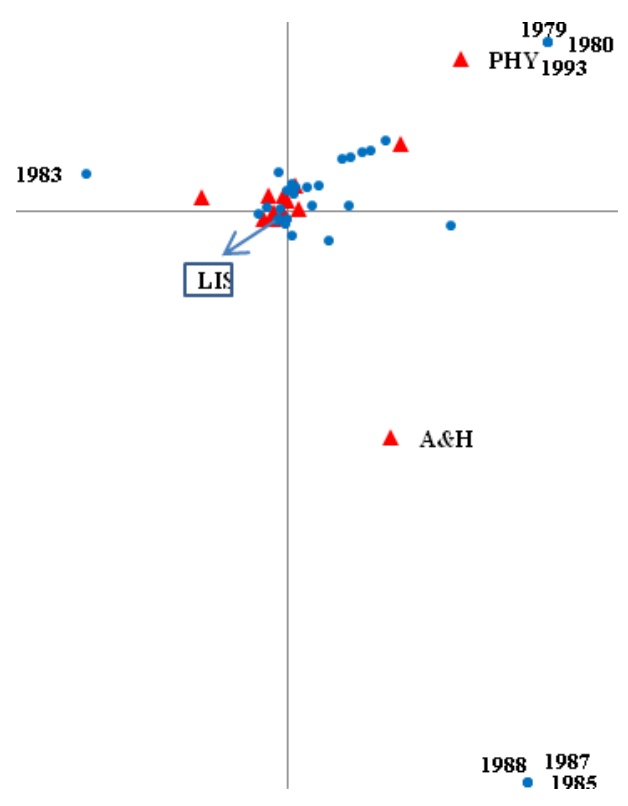

Fuente: WoS. Elaboración propia

Para poder visualizar las dos variables estudiadas, se ha ampliado el centro del mapa anterior en la figura 5. En ella se aprecia cómo, además de LIS, ocupan un lugar central Engineering (ENG) y Social Sciences (SSCC), a su vez los temas más frecuentes en los artículos de los autores españoles.

Figura 5 - Análisis de correspondencias año de publicación/área temática. (zona central del mapa). (España) 


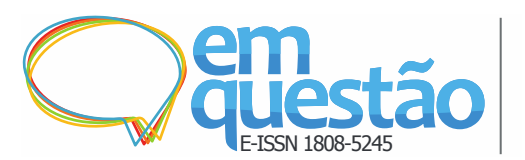

Los estudios métricos de información en Brasil y en España a partir de los artículos recogidos en la WoS María Luisa Lascurain

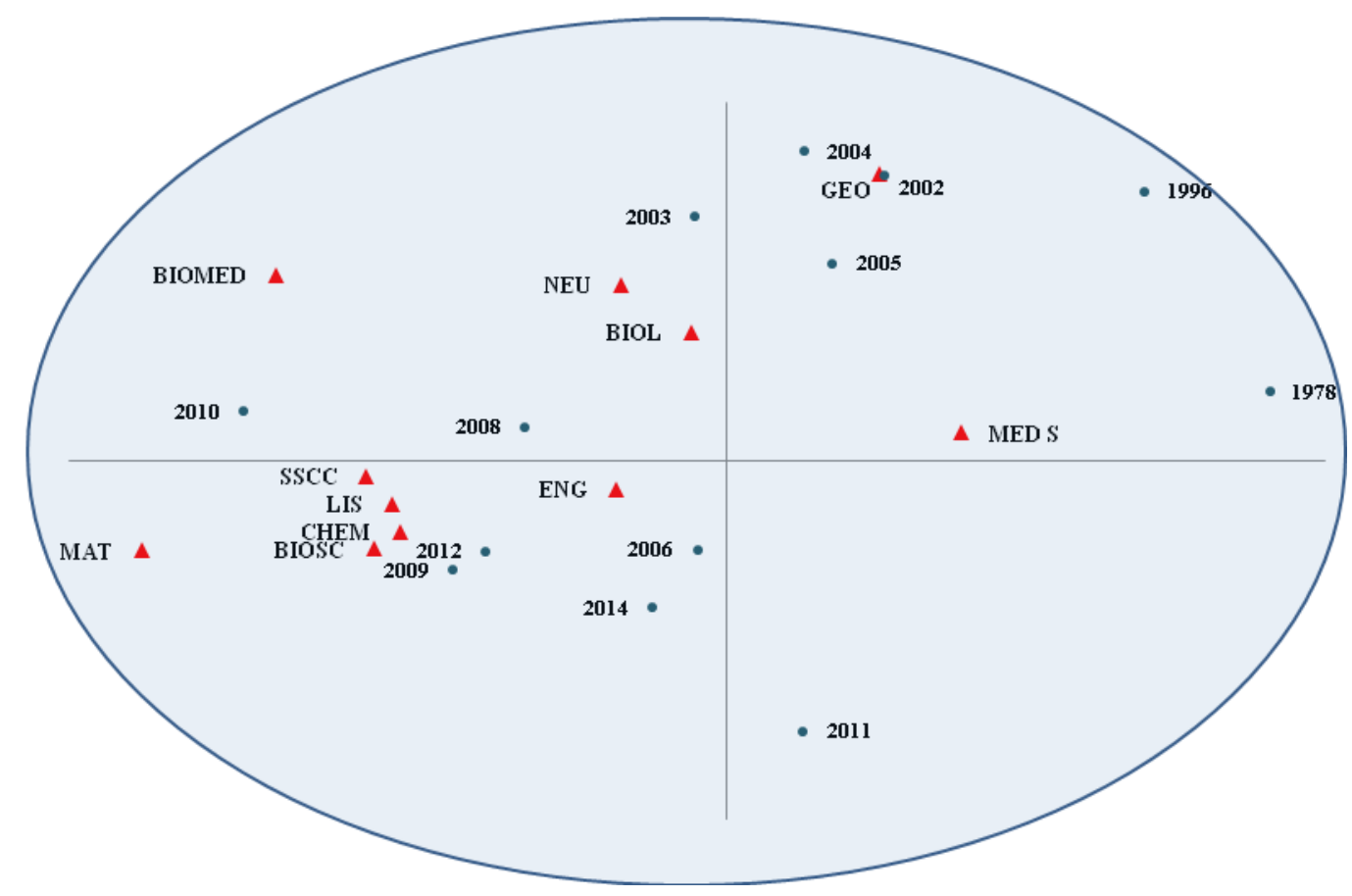

Fuente: WoS. Elaboración propia

En el mapa que relaciona las variables año y tema para los artículos de Brasil (figura 6), también encontramos próximos los temas más tratados por los autores del país junto con LIS, se trata de Engineering (ENG) y Medicine II (Non-Internal Medicine Specialties) (MED S). Un perfil más específico es el que comparten Physics (PHY) y Mathematics (MAT), ambas áreas con escaso número de registros. Social Sciences (CC SS) muestra su mayor aportación a finales del periodo estudiado.

Figura 6 - Análisis de correspondencias año de publicación/área temática (Brasil). 


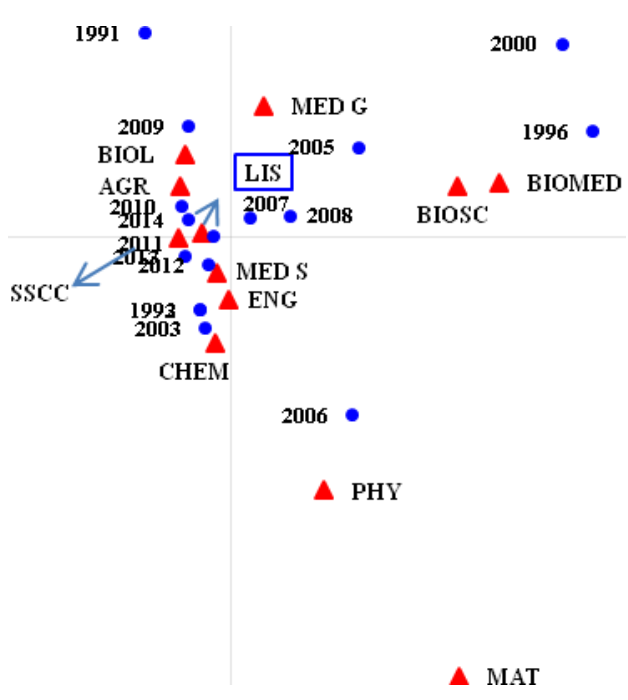

Fuente: WoS. Elaboración propia

\subsection{Dispersión de las publicaciones sobre EMI}

Los 744 artículos publicados por los autores españoles están recogidos en 244 títulos, mientras que los autores de Brasil publican los 219 artículos recuperados en 1994. La principal diferencias es que un $70 \%$ de las revistas elegidas por los investigadores españoles $(n=171)$ solo incluyen un trabajo sobre EMI, mientras que ese porcentaje es considerablemente menor en el caso de Brasil, ya que el $27 \%$ de los títulos tiene un solo artículo sobre el tema.

Siguiendo el modelo de Bradford para el cálculo de la dispersión de la literatura científica, encontramos que el $50 \%$ de los artículos españoles se publica en 14 títulos (el 5,74\%), mientras que para Brasil, este núcleo está formado por 11 revistas (el 11,70\%).

Los autores de ambos países elijen en primer lugar la revista Scientometrics para la publicación de su investigación sobre EMI, en concreto el $17 \%$, de los artículos publicados por los investigadores españoles $(n=126)$ y el 10,5\% ( $\mathrm{n}=23)$ de lo publicado por sus colegas de Brasil. Desde su fundación en Hungría en 1977, la revista constituye un importante foro de discusión sobre los estudios métricos de información. Otro tanto ocurre con Journal of Informetrics, 
la cuarta revista de publicación para los autores de España y la undécima para los brasileños, especializada en los aspectos cuantitativos de la información. Las dos clasificadas en el JCR en Information Science and Library Science.

A partir de aquí, la relación de revistas que conforman el núcleo no presenta coincidencias entre ambos países (Tabla 2).

Tabla 2 - Núcleo de revistas de publicación (España y Brasil)

\begin{tabular}{|c|c|c|c|}
\hline \multicolumn{2}{|l|}{ España } & \multicolumn{2}{|l|}{ Brasil } \\
\hline Revista & Artículos & Revista & Artículos \\
\hline Scientometrics & 126 & Scientometrics & 23 \\
\hline $\begin{array}{l}\text { Revista Española de } \\
\text { Documentación Científica }\end{array}$ & 58 & $\begin{array}{l}\text { Perspectivas em Ciência da } \\
\text { Informação }\end{array}$ & 19 \\
\hline El Profesional de la Información & 35 & Transinformação & 12 \\
\hline Journal of Informetrics & 27 & $\begin{array}{l}\text { Brazilian Journal of Medica } \\
\text { and Biological Research }\end{array}$ & 10 \\
\hline $\begin{array}{l}\text { Journal of the American Society } \\
\text { for Information Science }\end{array}$ & 25 & Revista de Saúde Pública & 10 \\
\hline Medicina Clínica & 21 & $\begin{array}{l}\text { Informação \& Sociedade. } \\
\text { Estudos }\end{array}$ & 9 \\
\hline Archivos de Bronconeumonía & 12 & $\begin{array}{l}\text { RAE-Revista de Administração } \\
\text { de Empresas }\end{array}$ & 6 \\
\hline Psicothema & 12 & $\begin{array}{l}\text { Anais da Academia Brasileira } \\
\text { de Ciências }\end{array}$ & 5 \\
\hline Research Evaluation & 11 & Cadernos de Saúde Pública & 5 \\
\hline Revista de Neurología & 11 & INTERCIÊNCIA & 5 \\
\hline Journal of Information Science & 10 & Journal of Informetrics & 4 \\
\hline $\begin{array}{l}\text { Information Research: An } \\
\text { Electronic Journal }\end{array}$ & 9 & & \\
\hline Investigación Bibliotecológica & 9 & & \\
\hline Nutrición Hospitalaria & 9 & & \\
\hline
\end{tabular}

En España, la mitad del núcleo lo forman revistas nacionales, si además se añade la presencia de la mexicana Investigación Bibliotecológica, el 57\% son además títulos en español. Ocho de las once revistas nucleares en el caso de Brasil son también nacionales y el portugués es el idioma del 72,73\%.

En cuanto a la clasificación temática que asigna el JCR a las revistas podemos comprobar, en consonancia con los resultados del análisis de los artículos, la diversidad de clasificaciones. En el núcleo de revistas sobre EMI, 
un porcentaje importante de títulos son de temas distintos de LIS: España tiene cuatro revistas de distintas especialidades de Medicina y una de Psicología, y Brasil cuenta con tres revistas de temas médicos y otras tres de temática Multidisciplinar, Ecología y Administración de empresas respectivamente.

\subsection{Colaboración España-Brasil en EMI}

El total de artículos publicados en colaboración España-Brasil es de nueve, lo que supone valores muy bajos en el total de la producción en ambos países (el $1,21 \%$ y el $4,11 \%$ respectivamente) siendo más representativa para Brasil. Este resultado es similar al encontrado por Moura et al.(2015) al analizar la producción conjunta de ambos países en la WoS.

Los trabajos fueron publicados entre 2007 y 2014, con una frecuencia de un artículo anual, excepto el año 2010 en que se publicaron dos.

El 44,44\% ( $n=4)$ ha sido publicado en portugués y el resto en inglés $(n=3)$ y en español $(n=2)$.

Como se recoge en la tabla 3, la dispersión de las revistas de publicación es alta, tratándose de revistas brasileñas $(n=4)$, españolas $(n=2)$ e internacionales $(n=2)$, considerando como tales las que no pertenecen a ninguno de los dos países estudiados.

Tabla 3 - Revistas de publicación de artículos en colaboración España-Brasil

\begin{tabular}{lc}
\hline Revista & Artículos \\
\hline Perspectivas em Ciência da Informação & 2 \\
Cadernos de Saúdee Pública & 1 \\
Informação \& Sociedade. Estudos & 1 \\
Journal of Informetrics & 1 \\
Nutrición Hospitalaria & 1 \\
Revista de Saúde Pública & 1 \\
Salud Mental & 1 \\
Scientometrics & 1 \\
\hline \multicolumn{2}{c}{ Fuente: WoS. Elaboración propia }
\end{tabular}

Los temas de estudio, según las categorías WoS aparecen recogidos en la tabla 4. La mitad de los mismos están clasificados en LIS y el resto en temas de Medicina y Salud. 
Tabla 4 - Temática de publicación (categoría WoS) de artículos en colaboración España-Brasil.

\begin{tabular}{lc}
\hline Categorías Wos & Frec. \\
\hline Information Science \& Library Science & 5 \\
Public, Environmental \& Occupational & \\
Health & 2 \\
Computer Science & 1 \\
Nutrition \& Dietetics & 1 \\
Psychiatry & 1 \\
\hline \multicolumn{2}{c}{ Fuente: WoS. Elaboración propia }
\end{tabular}

\section{Comentarios finales}

Uno de los aspectos a considerar en la consolidación de las disciplinas científicas es su inclusión en fuentes de referencia internacionales, como la Web of Science. En este sentido, los EMI se van afianzando como área de interés en España y en Brasil a juzgar por el incremento en el número de artículos sobre el tema a lo largo de 31 y 16 años respectivamente, con crecimientos (CMA) del $13,57 \%$ y del $25,17 \%$. Por una parte, el reconocimiento del papel de los estudios métricos de información como herramienta de evaluación de la ciencia y la tecnología y para la toma de decisiones en política científica y por otro lado la incorporación de revistas españolas y brasileñas a la WoS contribuyen a este hecho.

La publicación de trabajos en la WoS supone que los autores españoles y brasileños deben publicar en inglés, según los sesgos mencionados en el apartado de metodología. No obstante, el porcentaje de artículos en este idioma alcanza el 60\% aproximadamente, claramente inferior al encontrado por Moura et al. (2015) para el conjunto de publicaciones en colaboración España-Brasil que suponía entre el 80 y el $94 \%$.

En relación con la especialización temática, Information Science and Library Science (LIS) (como disciplina WoS y como área desagregada de Social Sciences según categorías Glänzel y Schubert) es la materia asignada a un mayor porcentaje de artículos tanto de Brasil como de España como cabía esperar. Además, el análisis de correspondencias entre los años de publicación y 
las categorías temáticas, muestra en los mapas obtenidos para los dos países la presencia de LIS a lo largo de todo el periodo estudiado.

Sin embargo, es preciso destacar el carácter interdisciplinar de los EMI: la búsqueda en la WoS se realizó en las tres bases de datos de la colección principal y sobre el campo tema (TS), ya que una acotación por LIS y por el SSCI dejaba fuera de la muestra un importante número de registros sobre estudios métricos. Es el caso de la segunda categoría WoS Computer Science o de Engineering, como área Glänzel y Schubert (en el 31,4 de los artículos españoles y en el 16,44\% de los de Brasil). En otras áreas temáticas las diferencias entre países permiten establecer perfiles diferenciados. En España destacan los estudios métricos relativos a distintas especialidades de la Medicina y de la Psicología, de gran tradición a partir de las publicaciones de López Piñero y Terrada en un caso y de Carpintero y sus discípulos (Tortosa y Peiró en la Universidad de Valencia entre otros) en el ámbito de la Historia de la Psicología (LASCURAIN SÁNCHEZ; LÓPEZ LÒPEZ; GONZÁLEZ UCEDA, 1997). En cuanto a la especialización temática de Brasil, además de los temas que comparte con España, el interés se centra en el ámbito de la Biología, la Investigación Biomédica y la Agricultura.

Esta interdisciplinariedad está presente asimismo en las revistas que eligen los autores para la difusión de su investigación. De las 14 revistas nucleares de publicación de los artículos españoles, un 35\% (n=4) están clasificadas en Medicina o Psicología, no en LIS. En el caso de Brasil, este porcentaje es superior ya que cerca de la mitad de las revistas pertenecen a otras clasificaciones del JCR (Multidisciplinar, Ecología y Administración).

En cuanto a la colaboración entre España y Brasil, los datos obtenidos muestran un escaso interés por este aspecto de la actividad científica que tantos beneficios aporta de cara a la visibilidad de las publicaciones y a su impacto y, en general, a la mejora cuantitativa y cualitativa de la investigación sobre los EMI. La presencia de solo nueve trabajos en colaboración no es suficiente para obtener unos perfiles de la misma y detectar puntos de interés común. Sin embargo, es de esperar que los intercambios académicos y de investigación que se vienen potenciando por parte de Universidades y grupos de los dos países 
redunden en un aumento de la cooperación que se refleje en publicaciones y proyectos de investigación.

\section{Referências}

ALCAÍN-PARTEARROYO, M. D. Aspectos métricos de la información científica. Ciencias de la Información, La Habana, n. 4, p. 32-36, 1991.

ARCHAMBAULT, E.; LARIVIERE, V. The limits of bibliometrics for the analysis of the social sciences and humanities literature. In: ARCHAMBAULT, E.; LARIVIERE, V. International social science council, world social sciences report: knowledge divides. Paris: UNESCO, 2010.

ARGUIMBAU-VIVÓ, L.; FUENTES-PUJOL, E.; GALLIFA-CALATAYUD, M. Una década de investigación documental sobre cienciometría en España: análisis de los artículos de la base de datos ISOC (2000-2009). Revista española de documentación científica, Madrid, v. 2, n. 36, p. 1-9, 2013.

ARQUERO AVILÉS, R.; OLIVÁN, S.; ANTONIO, J. Evaluación y análisis de la producción de los autores de trabajos difundidos en publicaciones periódicas editadas en español: Área de Biblioteconomía Documentación 1975-1984.

Documentación de las Ciencias de la Información, Madrid, n. 27, p. 53-74, 2004.

COLE, F. J.; EALES, N. B. The history of comparative anatomy. Science Progress, London, v. 11, p. 578-596, 1917.

DELGADO LÓPEZ-CÓZAR, E. La investigación en Biblioteconomía y Documentación. Gijón: Trea, 2002.

DELGADO LÓPEZ-COZAR, E. et al. Análisis bibliométrico y de redes sociales aplicado a las tesis bibliométricas defendidas en España (1976-2002): temas, escuelas científicas y des académicas. Revista Española de Documentación Científica, Madrid, v. 29, n. 4, p: 493-524, 2006.

DELGADOLÓPEZ-CÓZAR, E. et al. H Index Scholar: el índice h de los profesores de las universidades públicas españolas en humanidades y ciencias sociales. El profesional de la informacion, Barcelona, v. 23, n. 1, p. 87-89, 2014.

GARVEY, W.; GRIFFITH, B. Communication and information processing within scientific disciplines: empirical findings for psychology. Information Storage and Retrieval, Oxon, v. 8, p. 123-136, 1972. 
FRÍAS, J. A.; ROMERO GÓMEZ, P. ¿Quiénes son y qué citan los investigadores que publican en las revistas españolas de biblioteconomía y documentación? Anales de Documentación, Murcia, v. 1, p. 29-53, 1998.

GLÄNZEL, W.; SCHUBERT, A. A new classification scheme of science fields and subfields designed for scientometric evaluation purpose. Scientometrics, Amstderdam, v. 56, n. 3, p. 357-367, 2003.

GÓMEZ, I.; BORDONS, M. Limitaciones en el uso de los indicadores bibliométricos para la evaluación científica. Política Científica, [S.1.], n. 46, p. 21-26, 1996.

GORBEA-PORTAL, S. Tendencias transdisciplinarias en los estudios métricos de la información y su relación con la gestión de la información y del conocimiento. Perspectivas em Gestão \& Conhecimento, João Pessoa, v. 3, n. 1, p. 13-27, 2013.

HIDALGO-NUCHERA, A.; IGLESIAS-PRADAS, S.; HERNÁNDEZGARCÍA, Á. Utilización de las bases de datos de patentes como instrumento de vigilancia tecnológica. El profesional de la información, Barcelona, v. 18, n. 5, p. 511-519, 2009.

HULME, E. W. Statistical bibliography in relation to the growth of modern civilization. London: Grafton, 1923.

LASCURAIN SÁNCHEZ, M. L.; LÓPEZ LÒPEZ, P.; GONZÁLEZ UCEDA, L. Psicología y bibliometría en España. Revista General de Información y Documentación, Madrid, v. 7, n. 2, p. 95-107, 1997.

LÓPEZ-PIÑERO, J. M.; TERRADA, M. L. Veinte años de investigación bibliométrica en el Instituto de Estudios Documentales e Históricos sobre la Ciencia. Valencia: Universitat de València, 1993.

MOURA, A. et al. Panorama da produçao conjunta entre Brasil e Espanha indexada na WoS entre 2006-2012: indicadores de Atividade, Especialização e Colaboração. Informação \& Sociedade, João Pessoa, v. 25, n. 1, p. 76-82, 2015.

NACKE, O. Informetrie: ein neuer name für eine neue disziplin. Nachrichten Für Dokumentation, [S.1.], v. 30, n. 6, p. 219-226, 1979.

TORRES-SALINAS, D. et al. Clasificación integrada de revistas científicas (CIRC): propuesta de categorización de las revistas de ciencias sociales y humanas. El profesional de la Información, Barcelona, v. 19, n. 6, p. 675-683, 2010.

URBIZAGÁSTEGUI-ALVARADO, R.A. bibliometria no Brasil. Ciência da Informação, Brasília, v. 13, n. 2, p. 91-105, 1984. 
VANZ, S. A. S. A bibliometria no Brasil: análise temática das publicações do periódico Ciência da Informação (1972-2002). In: ENCONTRO NACIONAL DE PESQUISA EM CIÊNCIA DE INFORMAÇÃO, 5, 2003, Belo Horizonte. Anais.... Belo Horizonte: ANCIB, 2003.

\title{
Metric Studies of Information in Brazil and Spain seen from articles published in WoS
}

\begin{abstract}
This paper analyzes the articles on the disciplines that form the Metric Information Studies (EMI) in WoS that are signed by authors from Brazil and Spain. It begins with a very low number of documents, which increases over time, with a CMA higher for Brazil (25.17\%) than for Spain (13.57\%). The main language, with approximately $60 \%$ of the items, is English. The subjects, examined based in the WoS categories and in the grouping proposed by Glänzel e Schubert (2003), indicates the existance of areas other than LIS, such as Engineering or Medicine.The interdisciplinary approach is also evident in publication journals. The journal's core consists of national magazines $(72.73 \%$ for Brazil and 50\% for Spain). Collaboration between countries is very low, with only nine records throughout the period studied.
\end{abstract}

Keywords: Metric Studies of Information. Bibliometric Analysis. Brazil. Spain.

\section{Estudos métricos de informação no Brasil e na Espanha a partir dos artigos coletados na WoS}

Resumo: Este trabalho analisa a presença de artigos, assinados por autores do Brasil e da Espanha, sobre as disciplinas que formam os estudos métricos de informação (EMI) na WoS. Inicia-se com um número muito baixo de documentos, que cresce ao longo do tempo, com um CMA superior para o Brasil $(25,17 \%)$ do que para a Espanha $(13,57 \%)$. O idioma principal, com aproximadamente $60 \%$ dos artigos, é o inglês. A temática, examinada a partir das categorias WoS e da agrupação proposta por Glänzel e Schubert (2003), indica a existência de outras áreas diferentes à LIS, como Engenharia ou Medicina. A interdisciplinaridade dos trabalhos manifesta-se também nas revistas de publicação. Este núcleo está formado por publicações nacionais (72,73\% para Brasil e 50\% para Espanha). A colaboração entre países é muito escassa, com somente nove registros em todo o período estudado.

Palavras chave: Estudos métricos de informação. Análise bibliométrica. Brasil. Espanha. 
Los estudios métricos de información en Brasil y en España a partir de los artículos recogidos en la WoS María Luisa Lascurain

Recebido em 21/09/2015

Aceito em 17/12/2015

${ }^{1}$ MEIS, L.; LETA, J. O perfil da ciência brasileira. Rio de Janeiro: UFRJ, 1996. 